\title{
Evaluation of grey area mitigation tools within zonal and non-zonal RANS-LES approaches in flows with pressure induced separation
}

\author{
A. Probst ${ }^{\mathrm{a}, *}$, D. Schwamborn ${ }^{\mathrm{a}}$, A. Garbaruk ${ }^{\mathrm{b}}$, E. Guseva ${ }^{\mathrm{b}}$, M. Shur ${ }^{\mathrm{b}}$, M. Strelets ${ }^{\mathrm{b}}$, A. Travin ${ }^{\mathrm{b}}$ \\ a DLR, Center for Computer Applications in AeroSpace Science and Engineering, Göttingen, Germany \\ b St.-Petersburg Polytechnic University \& “New Technologies and Services" (NTS), St.-Petersburg, Russia
}

\section{A R T I C L E I N F O}

Article history:

Available online 5 September 2017

Keywords:

Hybrid RANS-LES

Wall-bounded flows

Pressure-induced separation

Grey-area mitigation

\begin{abstract}
A B S T R A C T
Hybrid RANS-LES computations of the separated flow over a wall-mounted hump are presented, which employ different grey-area mitigation techniques in the framework of a structured and an unstructured flow solver. Two zonal approaches using different synthetic-turbulence generators at the RANS-LES interface, as well as a non-zonal approach based on a shear-layer-adapted subgrid scale are compared in detail with validation data from a wind-tunnel experiment. Irrespective of the applied flow solver, the different methods are shown to be similarly effective in reducing the grey area compared to the basic hybrid RANS-LES model, and thus provide satisfying mean-flow predictions of the pressure-induced separation.
\end{abstract}

(c) 2017 Elsevier Inc. All rights reserved.

\section{Introduction}

Wall-bounded flows with non-fixed separation caused by an adverse pressure gradient (APG) feature several complex phenomena, such as turbulent boundary layer separation, reattachment of the separated shear layer, and recovery of the reattached turbulent boundary layer further downstream. These are common for many aerodynamic and industrial flows (e.g. wings/turbine blades near maximum loading) and present a serious challenge not only for RANS modelling but also for hybrid RANS-LES approaches. For the latter, the major difficulty in predicting such flows is associated with a strong delay of transition from modelled to resolved turbulence in the separated shear layers called the "grey area" issue (see Mockett et al., 2015a) resulting in a significant deviation of the predicted mean flow quantities and turbulence statistics from the experimental data. Note that the term "transition" refers here to a change of the modelling state (RANS vs. LES) of an already turbulent flow, and not to the classical "laminar-turbulent" transition. Although this difficulty is common to all hybrid RANS-LES methods, its origin and possible remedies are quite different for zonal and non-zonal methods.

In non-zonal ("DES-like") approaches, which rely on the natural instability of the separated shear layers, the delay of transition to developed 3D turbulence occurs because this instability is "blocked" by an excessive level of eddy viscosity in the initial region of the shear layers. This results from the convection of eddy

\footnotetext{
* Corresponding author.

E-mail address: axel.probst@dlr.de (A. Probst).
}

viscosity from the attached upstream boundary layer treated by RANS, as well as from a too strong generation of modelled turbulence in the separated region treated by LES. The latter is due to grid anisotropy (coarse in the spanwise and streamwise directions) typically used in this region and to the Smagorinsky-type subgrid modelling in classic DES, which was originally calibrated for developed 3D turbulence and therefore yields an overly large production term in 2D shear flow. Thus, in order to resolve the issue, one should ensure a considerable decrease of the eddy viscosity in the early shear layers (see, e.g. Shur et al., 2015, Mockett et al., 2015b).

For the zonal approaches, the issue results from a too slow transition from fully modelled turbulence in the upstream RANS zone to mostly resolved turbulence in the downstream LES zone (actually Wall-Modelled LES or WMLES zone). In this case, accelerating the transition process is only possible by improving the methods to inject artificial turbulence at the RANS-WMLES interface, which is a key element of zonal approaches.

Note finally that independently of the modelling approach, the efficiency of any grey-area mitigation tool is considerably affected by the grid resolution and the numerical discretization errors inherent to the flow solver. All these considerations have motivated the present study, aimed at comparing the performance of zonal and non-zonal hybrid RANS-LES approaches in different flow solvers for flows with APG-induced separation and reattachment.

The two considered zonal RANS-WMLES approaches employ the SST-based Improved Delayed Detached Eddy Simulation (IDDES) of Shur et al. (2008) in the WMLES zones, which are geometrically fixed throughout the simulation (thus, the term 'zonal'). The simulations performed differ both in the numerical solver and in the method used for the generation of turbulent content at the 

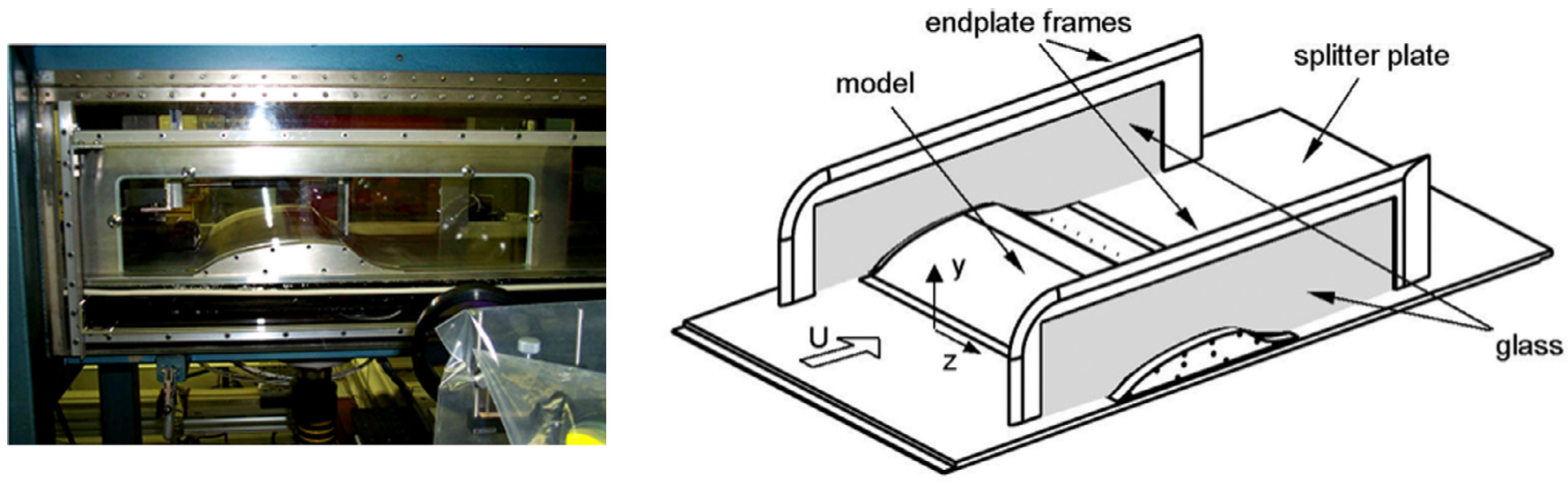

Fig. 1. Photo and sketch of the experimental setup.

RANS-WMLES interface. The first approach employs the unstructured DLR-TAU code (Schwamborn et al., 2008) with the Synthetic Eddy Method (SEM) by Jarrin et al. (2009), whereas the second one applies the Synthetic Turbulence Generator (NTS STG) developed by Shur et al. (2014) within the block-structured NTS inhouse code.

The non-zonal hybrid approach which is used only within the NTS code is the SST-based Delayed Detached Eddy Simulation (DDES) of Spalart et al. (2006) combined with the recently proposed shear-layer-adapted (SLA) definition of the subgrid lengthscale (Shur et al., 2015).

The performance of the three hybrid models is evaluated based on the 2D wall-mounted NASA hump test case (see Fig. 1), which had been purposefully designed as a CFD validation test case by Greenblatt et al. (2006). The key features of the flow (separation of the boundary layer from the hump, reattachment of the separated shear layer, and relaxation of the reattached boundary layer) are exactly the features we focus on in the present study.

\section{Numerical approaches}

The two research groups of DLR and NTS used their own numerical solvers and partly different physical-modelling approaches to simulate the common 2D hump test case. The following sections give a brief outline of these methods.

\subsection{Flow solvers}

DLR used its unstructured compressible finite-volume solver TAU, employing the recent implementations of the Synthetic-Eddy Method (SEM) by Jarrin et al. (2009) and the low-dissipation lowdispersion (LD2) scheme by Löwe et al. (2016). The latter is based on a 2nd-order energy-conserving skew-symmetric convection operator that is combined with a minimal level of 4th-order artificial matrix dissipation for stabilization. Moreover, the central flux terms employ an additional gradient extrapolation that effectively increases the discretization stencil and is used to reduce the dispersion error of the scheme. Both ingredients are essential for accurate WMLES results with the unstructured TAU code (Probst et al., 2016a). Note that in the present zonal RANS-WMLES computations, the LD2 scheme is only active in the respective WMLES region downstream of the interface. The temporal discretization is based on an implicit dual-time stepping scheme which is also of 2nd-order accuracy.

The simulations carried out by NTS were performed using the in-house NTS code, which is described in Shur et al. (2004). It is a cell-vertex finite-volume code accepting structured multi-block overset grids of Chimera type. The incompressible branch of the code employed here uses the flux-difference splitting method of Rogers and Kwak (1988). The approximation of the inviscid fluxes depends on the turbulence representation approach: in the zonal RANS-WMLES computations, it uses a 3rd-order upwind-biased scheme in the RANS zone and a 4th-order central scheme in the WMLES zone, whereas for the global DDES the hybrid (3rd-order upwind-biased/4th-order central) scheme of Travin et al. (2002) is used. The viscous fluxes are approximated with the 2nd-order central scheme. For the time integration, an implicit 2nd-order backward Euler scheme with sub-iterations is applied.

\subsection{Basic hybrid RANS-LES approaches}

The non-zonal simulation performed by NTS employs the Delayed Detached Eddy Simulation (DDES) of Spalart et al. (2006) which extends the classic Detached Eddy Simulation with a 'RANS shielding function'. This shielding function is designed to keep the hybrid length scale in attached boundary layers (e.g., upstream of the hump) in RANS mode $\left(l_{\text {hyb }} \approx l_{\text {RANS }}\right)$, and to allow LES mode $\left(l_{\text {hyb }} \approx l_{L E S}=C_{D E S} \Delta\right)$ only in separated or wake flows. While the subgrid filter width $\Delta$ in the original DDES is determined by the maximum local grid-edge length, $\Delta_{\max }$, it can be replaced by other filter definitions to tackle the grey-area problem (see Section 2.3).

For the zonal approaches with injection of synthetic turbulence, the hybrid RANS-LES methods need to be able to resolve the attached boundary layers upstream of separation. Both DLR and NTS achieve this by using the Improved Delayed Detached Eddy Simulation (IDDES) of Shur et al. (2008) which further extends the DDES by a wall-modelled LES (WMLES) branch in the hybrid length scale:

$l_{\text {hyb }}=\tilde{f}_{d} \cdot\left(1+f_{e}\right) \cdot l_{\text {RANS }}+\left(1-\tilde{f}_{d}\right) \cdot l_{\text {LES }}$.

Here, the blending function $\tilde{f}_{d}=\max \left\{\left(1-f_{d t}\right), f_{B}\right\}$ provides the mechanism to automatically switch between RANS $\left(f_{d t}=0\right)$, pure LES $\left(f_{d t}=1\right.$ and $\left.f_{B}=0\right)$, and WMLES $\left(f_{d t}=1\right.$ and $\left.0 \leq f_{B} \leq 1\right)$ modes, additionally involving a more complex filter-width definition to compute $l_{L E S}$. Note that in the zonal simulations of DLR, the WMLES mode is enforced by manually setting the shielding function $f_{d t}$ to 1 downstream of the pre-defined RANS-LES interface. However, this measure of precaution can be omitted without negatively affecting the results, as shown by the NTS simulations (see Section 4). Finally, the empirical "elevating" function $f_{e}$ increases the modelled Reynolds stress near the RANS-LES interface to ensure a continuous log-layer in WMLES (see Shur et al., 2008 for details).

All hybrid approaches chosen in the present study use the $k-\omega$ SST model of Menter (1994) as their RANS basis. 


\subsection{Grey-area mitigation tools}

As mentioned in Section 1, the DLR-TAU and NTS codes apply different methods for imposing turbulent content at a given RANSLES interface within zonal approaches.

The Synthetic Eddy Method (SEM) of Jarrin et al. (2009) used in the TAU code computes unsteady synthetic fluctuations, which recover the 1st- and 2nd-order statistical moments of the modelled (RANS) turbulence from upstream of the interface plane. To this end, a discrete set of vortex elements ('synthetic eddies') are randomly placed inside a rectangular box around the plane. These eddies are convected at bulk velocity through the box and are regenerated at the inlet upon exiting the box, thus keeping the total eddy number constant. Their sizes and intensities are derived from the RANS input statistics by employing a Cholesky decomposition of the Reynolds stress tensor. While the SEM is capable to recover all possibly anisotropy states of the input stress tensor, the synthetic velocity field is not divergence-free. The subsequent Divergence-free SEM of Poletto et al. (2011) remedies this potential drawback, but is not used in the present study. To transfer the induced velocity fluctuations into the actual flow simulation, TAU applies local forcing source terms in the momentum equations at the interface plane. A more detailed description of the SEM implementation in TAU can be found in Probst (2016b).

For their zonal simulations, NTS uses its own Synthetic Turbulence Generator (NTS STG) which is described in detail in Shur et al. (2014). This STG employs ideas of Kraichnan (1970) and has many common features with the STGs of Bechara et al. (1994), Smirnov et al. (2001), and Billson et al. (2003). It creates artificial ("synthetic") velocity fluctuations at the RANS-LES interface in the form of a superimposition of spatiotemporal Fourier modes with random amplitudes and phases. However, unlike other methods, it is capable of a plausible representation of the anisotropy of the vortical turbulent structures, which is an essential feature of nearwall turbulence. Other than that, it is free of the de-correlation issue inherent to many currently available STGs of the considered type. This is achieved thanks to the use of the global definitions of turbulence length and time scales involved in the wavelength scaling and by employing a set of wave numbers $\left\{k^{\mathrm{n}}\right\}$, which is fixed in time and over the entire RANS-LES interface. The latter ranges from the value corresponding to the largest wavelength for the considered problem up to the Nyquist limit. In addition, all the random quantities entering the STG are generated only once, at the beginning of the simulation (i.e., there are no random changes of phase, like in some other STGs). With the fixed set of wave numbers, this prevents generating unviable high-frequency "hash" which can lead to damping of the synthetic turbulence ("nearlaminarization") downstream of the interface.

While a fair comparison of the computational costs of SEM in STG is complicated by the use of two rather different codes and hardware setups, it can be stated for both methods that the additional time effort to generate the synthetic fluctuations in the present test case is less than $0.5 \%$ of the respective computing time for a whole physical time step.

For the non-zonal DDES computations of NTS, in order to decrease a strong delay of RANS-to-LES transition in the separated shear layer (cf. Fig. 2), a shear-layer-adapted definition of the subgrid length scale ("filter size"), $\Delta_{S L A}$, is used. This formulation combines two approaches to reduce the excessive eddyviscosity levels in the initial quasi-2D shear layers mentioned in Section 1: first, a vorticity-sensitive grid-filter size adopted from Mockett et al. (2015b) is used, which diminishes the impact of the commonly large grid anisotropy in the direction orthogonal to the 2D flow plane. Second, an empirical function for "unlocking" the Kelvin-Helmholtz instability, which is based on a kinematic measure for the two-dimensionality of the flow ("vortex tilting measure"), is applied to further scale down the grid filter (and thus the eddy viscosity) in quasi-2D flow. A detailed outline of the physical background behind this definition, its formulation, and description of the corresponding DDES version are given in Shur et al. (2015) and Guseva et al. (2017).

\section{Test case description and simulation setup}

The NASA wall-mounted hump test case of Greenblatt et al. (2006) has already been used in numerous computational studies, including those carried out in the framework of specially organized workshops, as well as in the course of the EU projects ATAAC (Schwamborn and Strelets, 2012) and Go4Hybrid (Mockett et al., 2015a). As a purposefully designed validation case for numerical simulations, it has proven a challenging case for both RANS and hybrid RANS-LES methods. As an example, Fig. 2 demonstrates the unsatisfactory prediction of the skin-friction distribution over the hump obtained with the Spalart-Allmaras (SA) and SST RANS models (Spalart and Allmaras, 1994; Menter, 1994), as well as SA- and SST-based DDES employing the standard maximum edge-length definition of the subgrid scale, $\Delta_{\max }$.

The hump chord length, $c$, in the experiments is equal to $0.42 \mathrm{~m}$, and the crest height is $0.0537 \mathrm{~m}$. The characteristic Reynolds number based on the chord is about $10^{6}$ and the Mach number is 0.1 , which allows both incompressible and compressible simulations.

The computational domain in the $X Y$-plane is shown in Fig. 3. Its size in the spanwise direction is equal to $0.4 c$, which has been proven sufficient to arrive at a span-independent solution in the ATAAC project. As recommended on the Turbulence Modeling Resource Portal handled by NASA (https://turbmodels.larc.nasa.gov/), the contour of the upper (slip) wall of the domain was modified (moved downwards) in the area above the hump in order to compensate the blockage effect of the endplates (see Fig. 1).

The inflow and outflow conditions in the DLR and NTS simulations were somewhat different since DLR used the compressible TAU code and NTS employed the incompressible branch of the NTS code. In both cases velocity and turbulent quantities at the inflow boundary $(x / c=-2.14)$ were specified from a precursor SST RANS of the zero pressure gradient boundary layer, carried out to match the experimental value of the momentum thickness based $R e$-number $\operatorname{Re}_{\theta_{\text {inflow }}}=\theta_{\text {inflow }} U_{\infty} / \nu=7200$. In the incompressible simulations (NTS), the inflow static pressure was defined by the linear extrapolation of the values in the nearby interior cells of the domain, whereas in the compressible simulations (DLR) flux boundary conditions were used together with an extrapolation of the pressure from the interior. At the outflow boundary, NTS specified a constant static pressure and defined all the other flow variables by linear extrapolation from the interior of the domain, which is similar to DLR's flux conditions with an imposed fixed exit pressure. Finally, in all simulations the boundary conditions in the spanwise direction were periodic.

The grid used in all the simulations is shown in Fig. 4. It has $511 \times 127$ cells in the $x$ - and $y$-directions, respectively. Near the bottom wall, the grid is wall-orthogonal and yields a first cell height in wall units $\left(\Delta y_{1}^{+}\right)$of less than 1.0 in the whole domain. For $-1.0<x / c<0.5$, the grid is also refined in the $x$ direction in order to allow placing the RANS-LES interface at any cross-section within this region. In the separation ("focus") region $(0.67<x / c<1.5)$ both $x$ - and maximum $y$-steps are equal to $0.005 c$. In spanwise direction, the grid has 80 cells and is uniform with a step size equal to $0.005 c$, thus resulting in a nearly cubic grid in the focus region. The total grid size is around 5.2 million cells. 

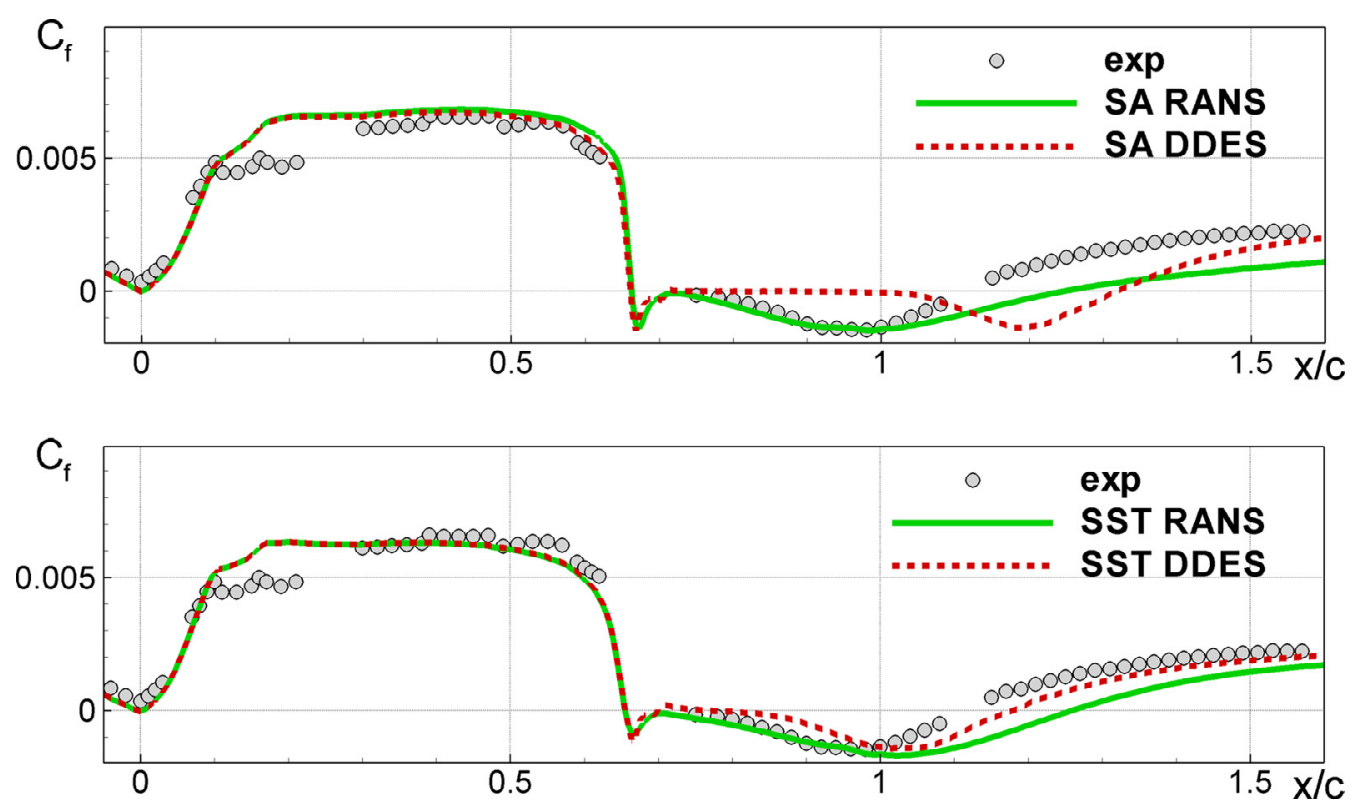

Fig. 2. SA and SST RANS and SA- and SST-based DDES predictions of skin-friction coefficient distribution over the 2D hump flow.

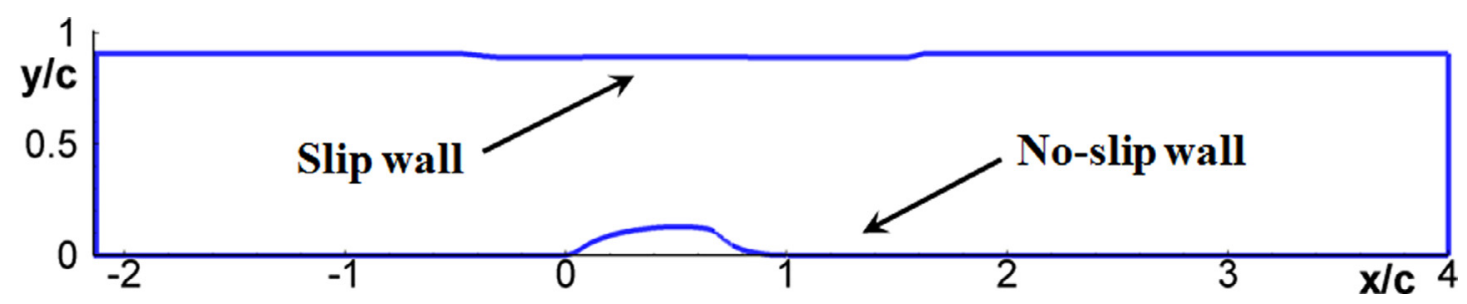

Fig. 3. Schematic of computational domain in $X Y$-plane.
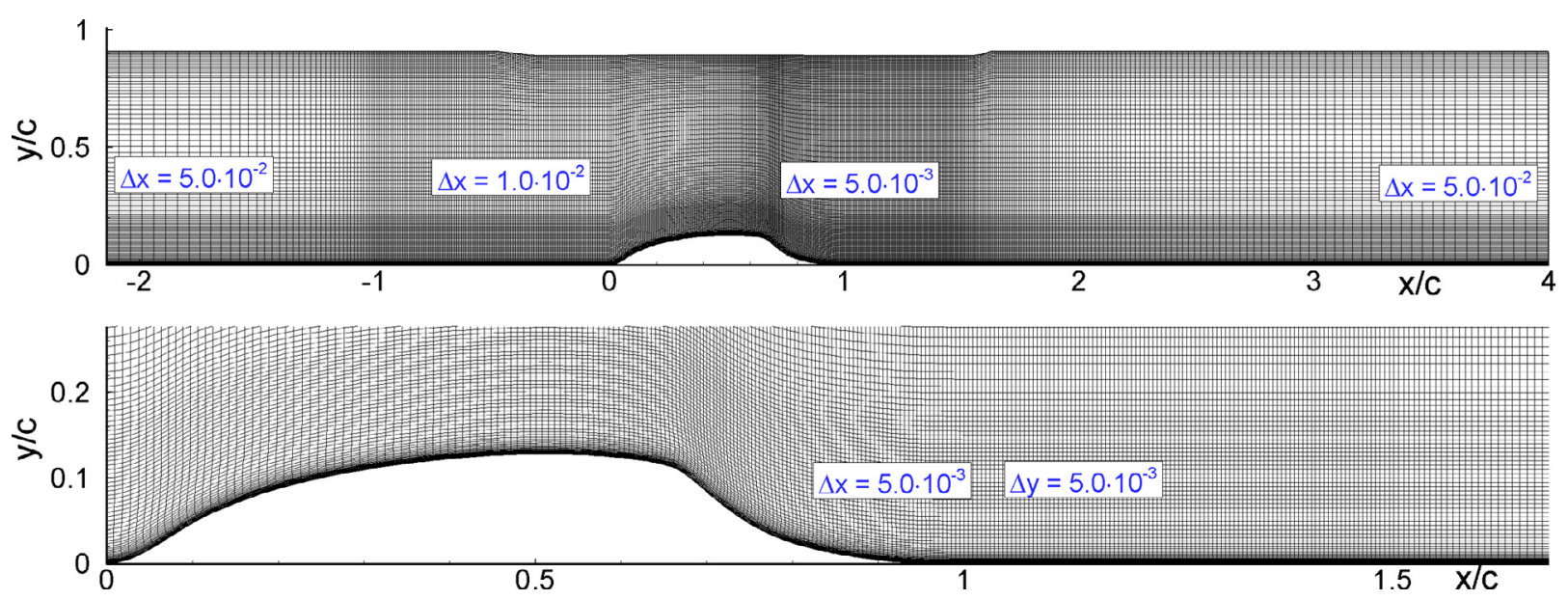

Fig. 4. Full grid in $X Y$-plane used in the simulations and its zoomed in view.

In all simulations, the physical time step is set to $\Delta t=0.002 c / U_{\infty}$ which results in a (convective) CFL number of well below 0.5 in the focus region. The total simulation times vary around 30 convective time units $\left(1 \mathrm{CTU}=c / U_{\infty}\right)$, where the last 20 CTU are used for obtaining statistically-averaged flow data.

\section{Simulation results}

Five full hybrid RANS-LES computations of the 2D hump flow were conducted for the present evaluation. Table 1 summarizes their respective primary settings.

\subsection{Qualitative assessment}

Figs. 5 and 6 show flow visualizations illustrating a comparative qualitative performance of the three modelling approaches considered in the present study. Their analysis allows drawing the following conclusions.

First, the figures suggest that both zonal approaches ensure rapid formation of developed $3 \mathrm{D}$ turbulence downstream of the RANS interface independently of its location. However, turbulence structures in the close vicinity of the interface in the DLR and NTS simulations look somewhat different (compare frames "a" and "b" in Figs. 5 and 6), which is not surprising considering the differ- 
Table 1

Matrix of simulations performed.

\begin{tabular}{|c|c|c|c|c|c|}
\hline Partner & Approach & Scale-resolving method & Grey-area mitigation & RANS-LES interface location & CFD code \\
\hline DLR & Zonal & SST-based IDDES & SEM & $\begin{array}{l}x / c=0.5 \\
x / c=-1.0\end{array}$ & Compressible TAU code with LD2 scheme \\
\hline NTS & Zonal & SST-based IDDES & NTS STG & $\begin{array}{l}x / c=0.5 \\
x / c=-1.0\end{array}$ & Incompressible branch of NTS code \\
\hline NTS & Non-zonal & SST-based DDES & Shear-layer-adapted length scale & - & Incompressible branch of NTS code \\
\hline
\end{tabular}
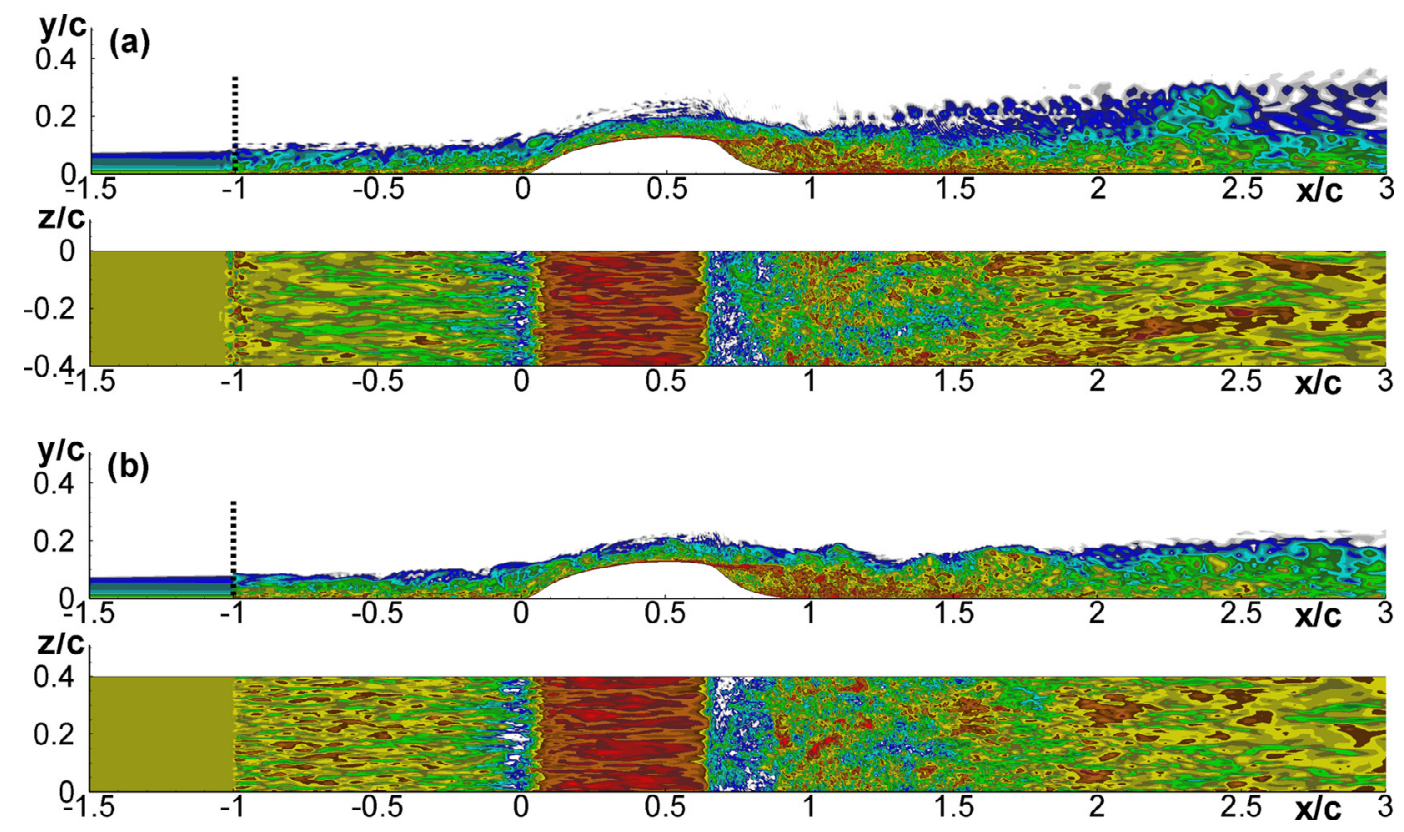

Fig. 5. Snapshots of vorticity magnitude in $X Y$-plane and on bottom wall from zonal RANS-IDDES with interface at $x / c=-1.0$. (a) DLR; (b) NTS.

ent turbulence generators used in these simulations. At the same time, further downstream no tangible qualitative difference of the resolved turbulence is observed. Hence, judging by the visualizations of the considered flow, SEM and NTS STG within both CFD codes ensure rather short relaxation lengths.

Second, both zonal simulations indicate a resolution of fine turbulent structures compatible with the cell sizes of the computational grid in the "focus" region downstream of the separation point, thus suggesting that the unstructured TAU code using the LD2 scheme provides low numerical dissipation comparable with that of the higher-order structured NTS code.

Finally, a comparison of the flow visualizations from the zonal IDDES (Figs. 5 and 6a, b) and global DDES with $\Delta_{\text {SLA }}$ (Fig. 6c) shows that downstream of the separation point both approaches return similar solutions. In particular, they both ensure a rapid break-up of the separated shear layer and transition to developed 3D turbulence. In contrast to this, the original DDES with $\Delta_{\max }$ (Fig. 6d) reveals a considerable delay of formation of the resolved 3D turbulent structures in the separated shear layer. This supports a high efficiency of the $\Delta_{S L A}$ definition of the subgrid length scale.

\subsection{Validation of mean-flow predictions}

The computed mean surface flow characteristics (coefficients of skin friction, $C_{f}$, and wall pressure, $C_{p}$,) are compared with each other and with the experiment in Figs. 7 and 8. In line with expectations based on the flow visualizations in Section 4.1, these predictions turn out to be very similar. On a global scale, they all agree well with the experimental data, which supports quantitative accuracy of the simulations. In particular, Figs. 7c and $8 c$ demonstrate a comparable performance of the non-zonal DDES $+\Delta_{S L A}$ to the zonal approaches (the radical improvement of the DDES accuracy thanks to the $\Delta_{S L A}$ subgrid scale is seen from a comparison of Figs. 7c and 2).

Closer inspection reveals some subtle differences between the simulation results. While the NTS STG induces a notable, but only short drop of skin friction just downstream of the respective interface (Fig. 7a and b), the DLR SEM results yield a more continuous $C_{f}$ evolution. However, with the SEM interface at $x / c=-1$ the $C_{f}$ value gradually falls below its initial (RANS) level over a considerable distance before recovering at around $x / c=-0.2$ to the curves from the other simulations. In absence of experimental data in this region, the results from local RANS modelling in Fig. 7b are considered as sufficiently accurate reference. Although this initial underprediction in the WMLES region cannot be clearly confirmed for the DLR simulation with the downstream interface $(x / c=0.5)$, it may be associated with the slightly larger deviation from the experiment around reattachment in Fig. 7b. Accordingly, DLR's reattachment locations summarized in Table 2 show a larger sensitivity to the interface location than the zonal computations of NTS.

As for the non-zonal DDES with $\Delta_{S L A}$, the skin-friction distribution is naturally free from local disturbances due to syntheticturbulence injection. However, it yields a more pronounced $C_{f}$ drop than the zonal approaches just after separation $(x / c \approx 0.66)$ along with a slightly over-predicted separation zone (Fig. 7c) which suggests to be a remainder of the grey area issue.

Similar conclusions can be drawn from the comparison of the predicted streamwise velocity profiles at different cross sections with corresponding experimental data in Fig. 9, although at $0.65 \leq x / c \leq 0.9$ these profiles turn out to be somewhat more sen- 

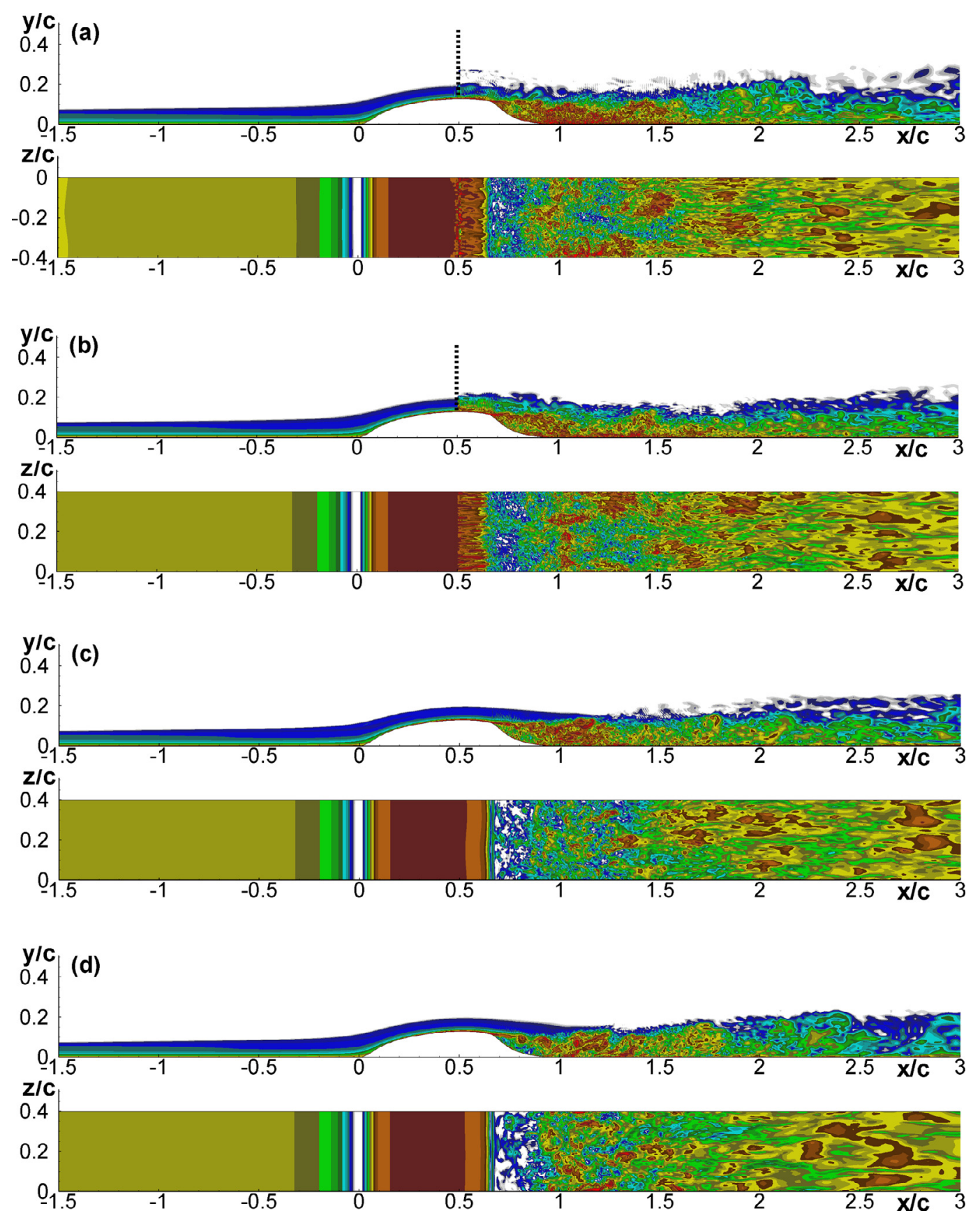

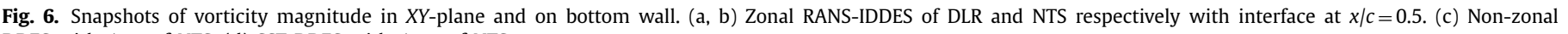
DDES with $\Delta_{S L A}$ of NTS. (d) SST DDES with $\Delta_{\max }$ of NTS.

Table 2

Comparison of separation and reattachment locations from different simulations with experiment.

\begin{tabular}{|c|c|c|c|c|c|}
\hline Code & Approach & $\begin{array}{l}\text { Grey-area } \\
\text { mitigation }\end{array}$ & $\begin{array}{l}\text { RANS-LES } \\
\text { interface location }\end{array}$ & $\begin{array}{l}\text { Separation } \\
\text { point, } x / c\end{array}$ & $\begin{array}{l}\text { Reattachment } \\
\text { point, } \mathrm{x} / \mathrm{c}\end{array}$ \\
\hline \multirow[t]{2}{*}{ DLR-TAU } & Zonal IDDES & SEM & $x / c=0.5$ & 0.655 & 1.152 \\
\hline & & & $x / c=-1.0$ & 0.656 & 1.114 \\
\hline \multirow[t]{2}{*}{ NTS } & Zonal IDDES & NTS STG & $x / c=0.5$ & 0.656 & 1.131 \\
\hline & & & $x / c=-1.0$ & 0.658 & 1.120 \\
\hline NTS & Non-zonal DDES & Shear-layer-adapted length scale & - & 0.656 & 1.143 \\
\hline Uzun and Malik (2017) & Wall-resolved LES & - & - & 0.659 & 1.095 \\
\hline Experiment & & & & 0.665 & 1.11 \\
\hline
\end{tabular}



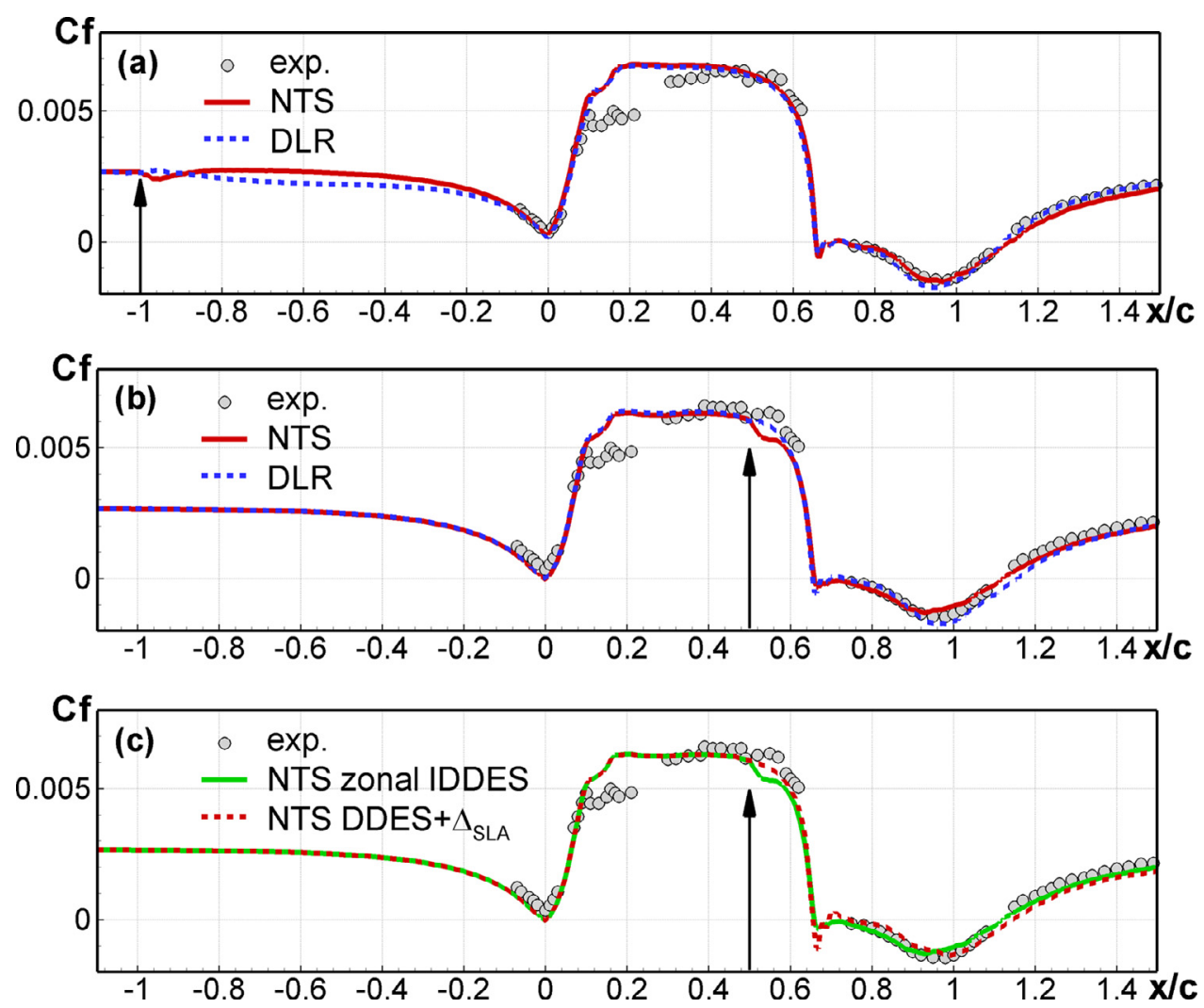

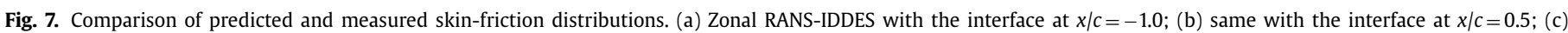

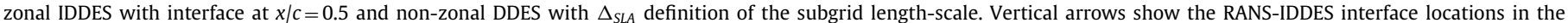
zonal simulations.
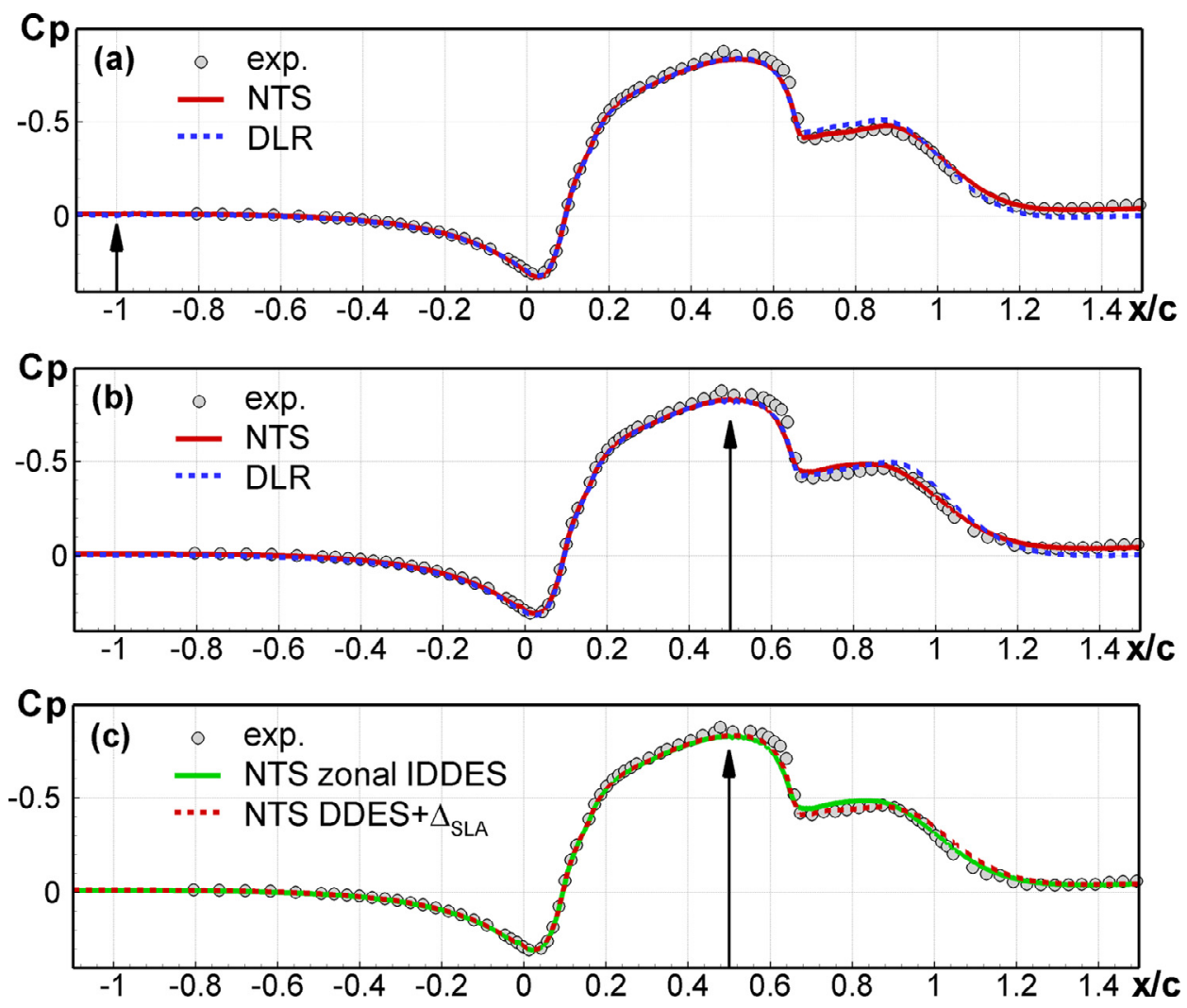

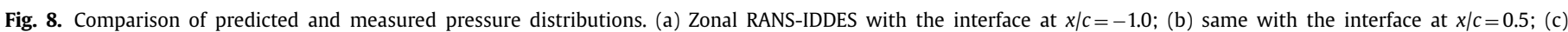

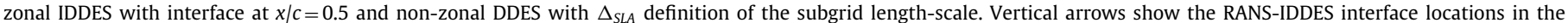
zonal simulations. 

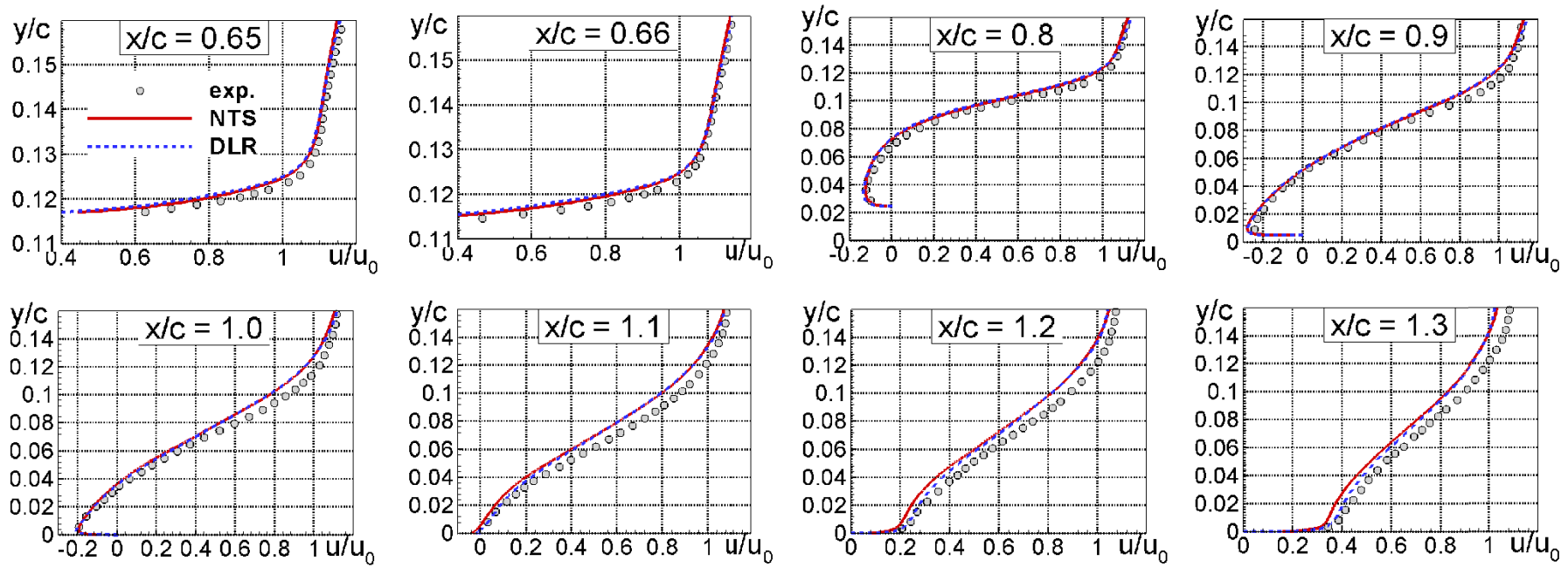

(a) Zonal RANS - IDDES with interface located at $\mathrm{x} / \mathrm{c}=-1.0$
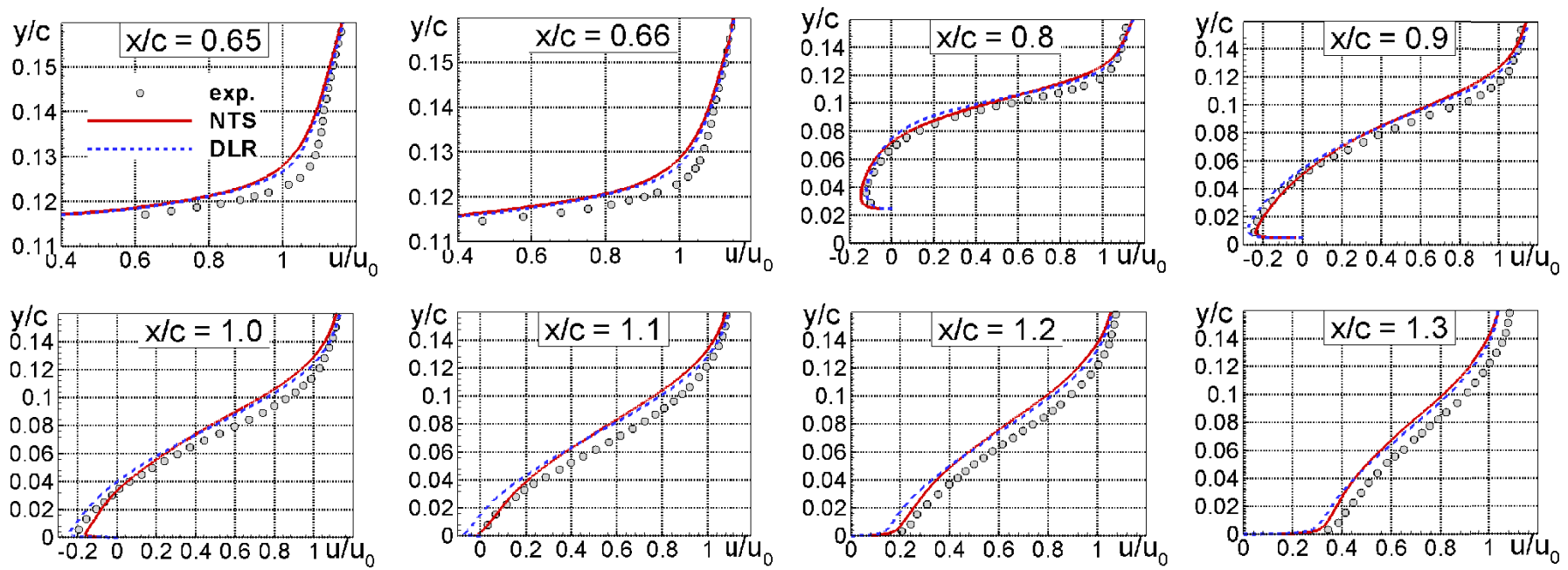

(b) Zonal RANS - IDDES with interface located at $\mathrm{x} / \mathrm{c}=0.5$
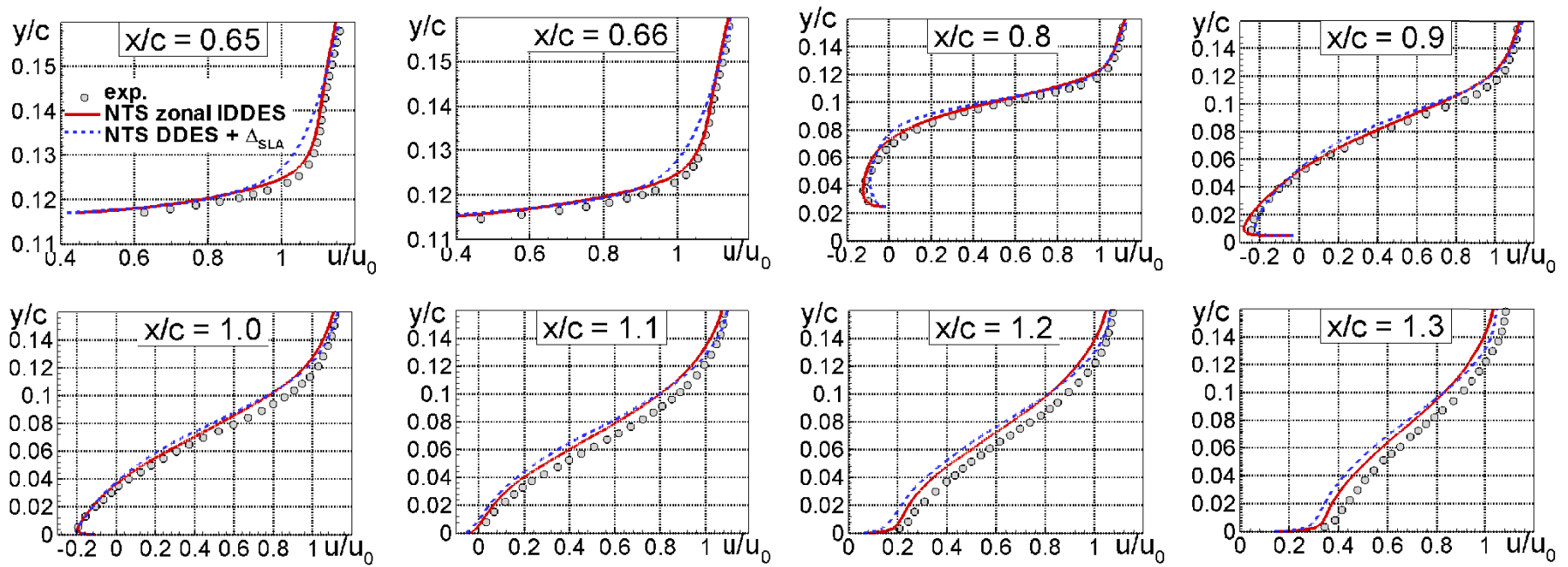

(c) Non-zonal DDES with $\Delta_{S L A}$ subgrid scale and zonal RANS - IDDES with interface located at $\mathrm{x} / \mathrm{c}=-1.0$

Fig. 9. Comparison of predicted and measured streamwise velocity profiles at different flow cross-sections. 

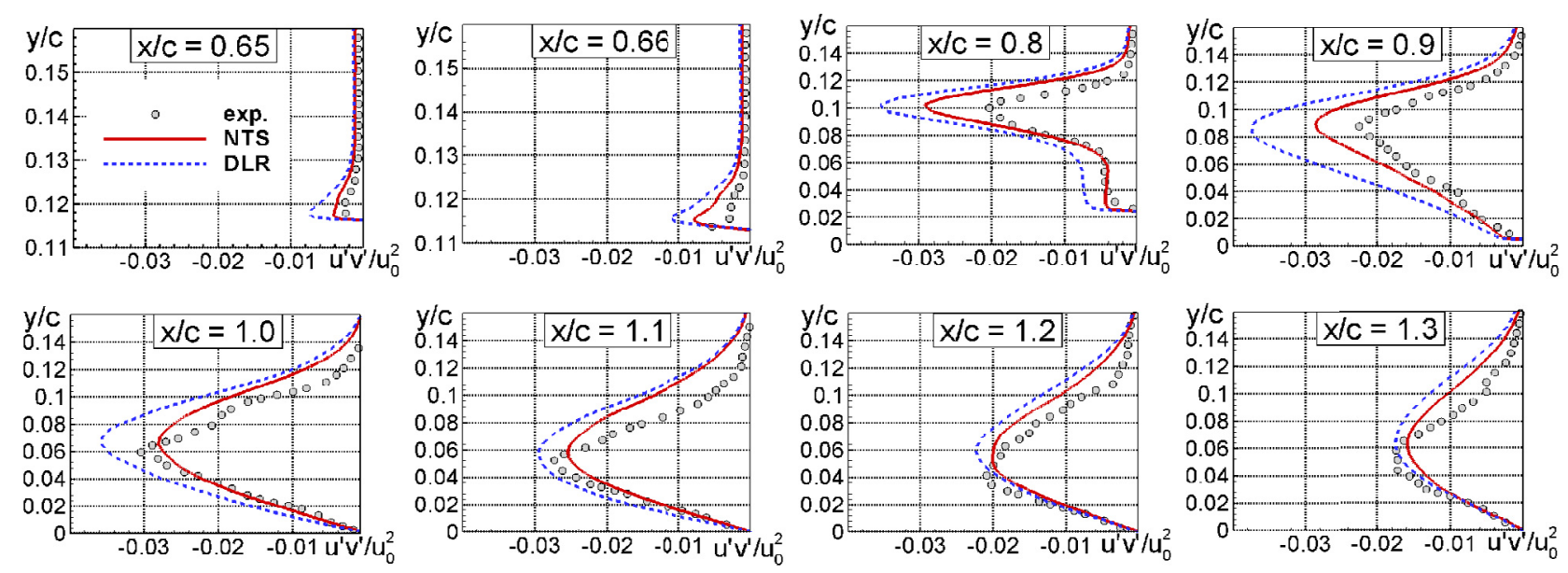

(a) Zonal RANS - IDDES with interface located at $\mathrm{x} / \mathrm{c}=-1.0$
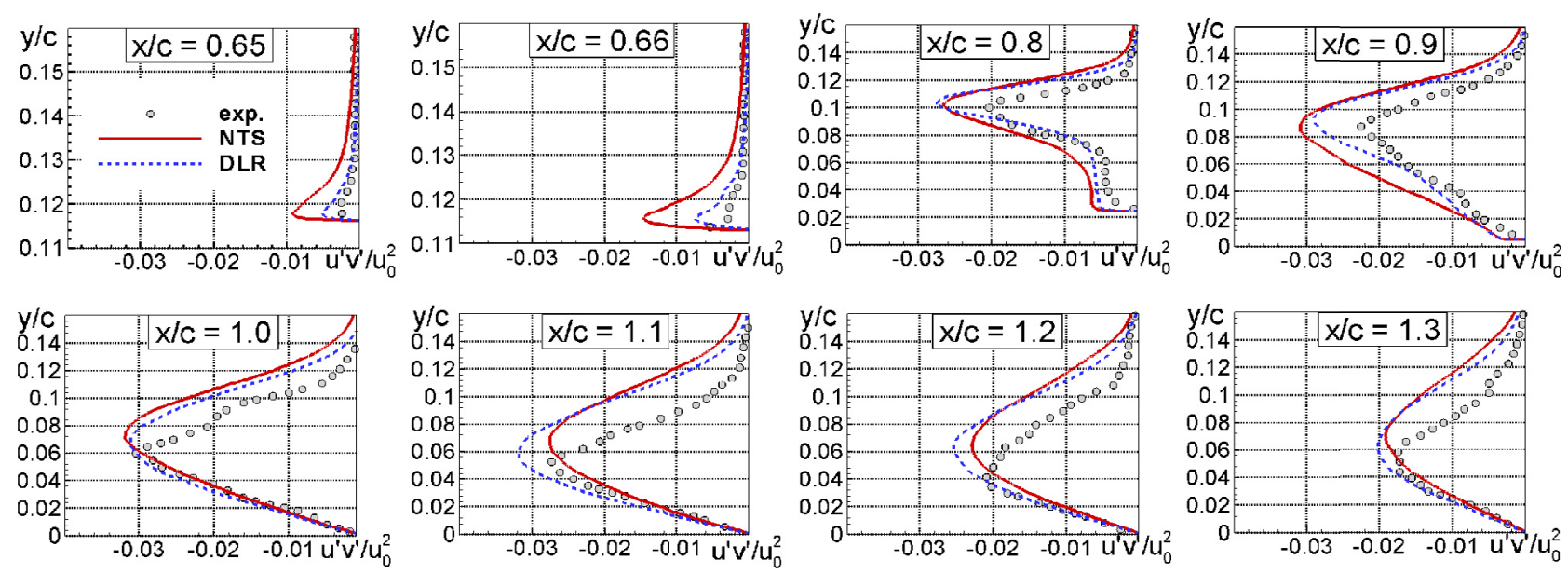

(b) Zonal RANS - IDDES with interface located at $\mathrm{x} / \mathrm{c}=0.5$
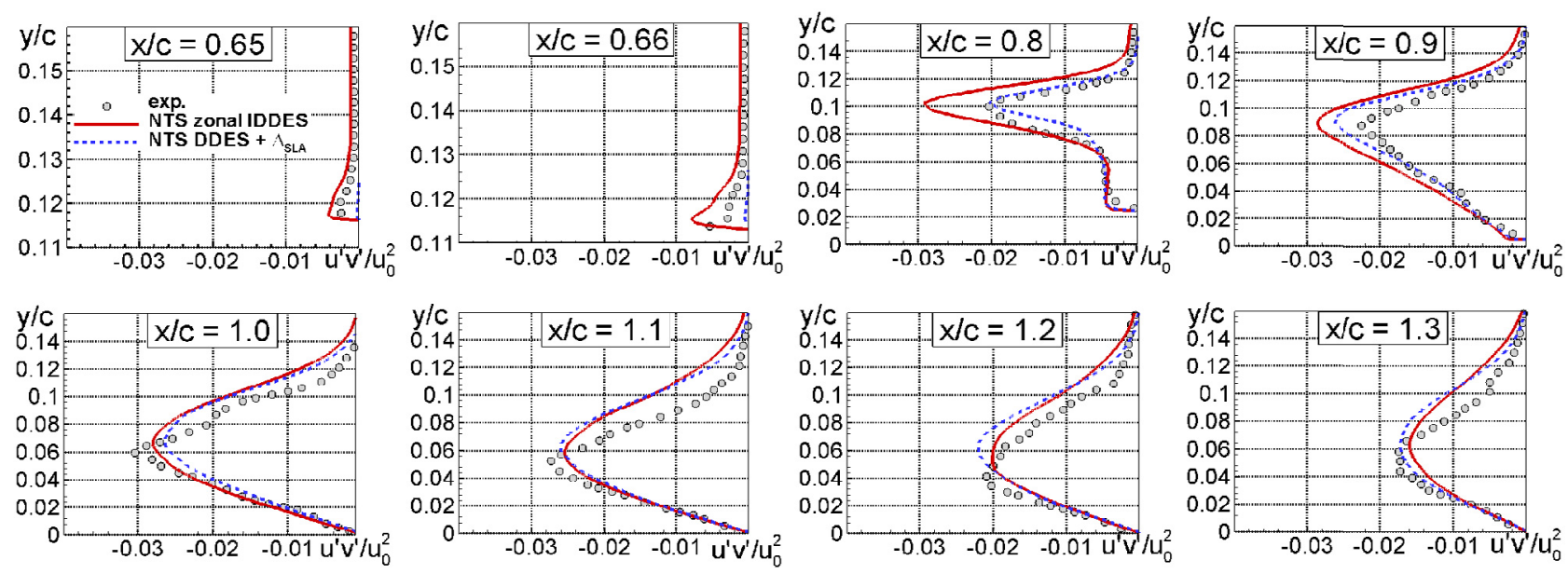

(c) Non-zonal DDES with $\Delta_{\text {SLA }}$ and zonal RANS - IDDES with interface located at $x / c=-1.0$

Fig. 10. Comparison of predicted resolved and measured Reynolds shear stress profiles at different flow cross-sections. 
sitive to the location of the RANS-IDDES interface than the $C_{f}$ and, especially $C_{\mathrm{P}}$ distributions.

In the simulations with the interface located at $x / c=0.5$ the difference between the DLR and NTS predictions becomes a bit larger than for the interface at $x / c=-1.0$, which is best seen by comparing the profiles at $x / c=1.0-1.2$ in Fig. $9 a$ and $b$. This is mainly due to the larger sensitivity of the DLR results to the interface location that was already observed in Fig. 7.

Placing the interface farther upstream of the hump (at $x / c=-1.0$ ) ensures overall somewhat better agreement with the experiment than placing it at $x / c=0.5$. In particular, the two profiles just around the separation point $(x / c=0.65$ and 0.66$)$ are best predicted with the far-upstream interface (Fig. 9a), whereas the downstream interface (Fig. 9b) and the non-zonal DDES (Fig. 9c) yield a slightly too large momentum loss in this critical region.

Moreover, all simulations tend to over-predict the near-wall momentum loss around reattachment and recovery to a different degree. In line with the mean surface data in Figs. 7 and 8, the non-zonal DDES with $\Delta_{S L A}$ yields slightly larger deviations from the experiment than the zonal computation at all considered profile locations, see Fig. 9c.

Nonetheless, taking all uncertainties with respect to the measurement and the simplified simulation setup into account, all these discrepancies are insignificant, and the predictions of the mean velocity profiles by all the considered simulations may be assessed as fairly accurate. This is supported also by Table 2, where we present coordinates of the separation and reattachment points predicted by all the considered approaches with each other, with the experimental data, and with predictions of the wall-resolved LES with the Vreman subgrid scale model carried out by Uzun and Malik (2017) on a grid with around 400 million points.

Finally, Fig. 10 compares the resolved shear stress profiles predicted by the zonal and non-zonal hybrid approaches with the total measured Reynolds stress (note that such a comparison is justified because the input of the modelled stresses in the zonal simulations is negligible, while in the non-zonal simulation it is considerable only in the early separated shear layer).

As seen in Fig. 10a, for the interface located at $x / c=-1.0$, both zonal approaches overestimate the experimental peak values of the stress within the range $0.65 \leq x / c \leq 0.9$, the difference being somewhat larger in the DLR simulations. Further downstream $(x / c \geq 1.0)$ the discrepancy between the simulations and the experiment, as well as between the two simulation approaches decreases.

With the interface location at $x / c=0.5$ (Fig. 10b), the difference between the two considered approaches becomes somewhat smaller, except for the very beginning of the separated shear layer $(x / c=0.65$ and 0.66$)$, where the NTS predictions strongly overestimate the peak stress values. However, due to the strong sudden pressure rise visible in Fig. 8, the location of separation is virtually not affected by this discrepancy (see Table 2). At the same time, as could be expected, the agreement of the simulations with the experiment becomes somewhat worse compared to the simulation with the interface at $x / c=-1.0$.

Finally, Fig. 10c compares performance of the zonal RANS-IDDES approach of NTS and non-zonal DDES combined with the $\Delta_{\text {SLA }}$ definition of the subgrid length scale.

As already indicated above, a delay of transition to resolved 3D turbulence does exist in the non-zonal DDES: in this simulation, the resolved stress at the first two flow cross-sections $(x / c=0.65$ and 0.66 ) is close to zero. However, further downstream it rapidly becomes comparable with the stress predicted by the zonal simulation. Thus, the figure suggests that the use of the shear-layeradapted definition of the subgrid length scale is an efficient grey area mitigation tool, which makes DDES competitive with the zonal approaches.

\section{Conclusions}

Results of the simulations of the 2D NASA wall-mounted hump flow carried out with two classes of grey-area mitigation techniques (zonal RANS-IDDES with synthetic turbulence and nonzonal DDES combined with the shear-layer-adapted subgrid length scale) allow drawing the following conclusions.

For the considered flow, both grey area mitigation tools are proven to be nearly equally effective in terms of decreasing the delay of transition from the fully modelled (RANS) to mostly resolved (LES) turbulence in the separated shear layer. This, in turn, leads to a drastically improved agreement with the experiment compared to the standard non-zonal SA-DDES approach, in which the delay is strongly pronounced.

Along with this, the results of the simulations suggest that the zonal RANS-IDDES technique implemented in two rather different industrial codes (the unstructured DLR-TAU code using the LD2 scheme and the multi-block structured code of NTS) provide close predictions, thus supporting the robustness of the approaches. Apart from minor deviations and a slightly larger sensitivity of the DLR approach to the interface location, no substantial difference is found in the performance of the two methods used for creating turbulent content at the RANS-IDDES interface, namely, the SEM of Jarrin et al. (2009) and NTS STG of Shur et al. (2014).

These findings are rather favourable and give solid evidence of the robustness and efficiency of both types of grey-area-mitigation tools. Note however that the considered test case is characterized by a nearly fixed separation, which makes it not very sensitive to the accurate resolution of the attached turbulent boundary layer prior to separation. Therefore, in the more severe test cases with non-fixed separation from a smooth body caused by either adverse pressure gradient or shock / boundary layer interaction, the performance of the considered approaches may be not that successful. This concern is supported by a recent study of Spalart et al. (2016) on zonal RANS-IDDES simulations of the Bachalo-Johnson transonic bump flow (Bachalo and Johnson, 1986) with the use of the NTS STG, who found that the approach fails to predict the shock location and other flow characteristics with sufficient accuracy. Although some flaws of the IDDES as a wall model for WMLES probably explain this failure better than imperfections of the NTS STG, this suggests the necessity for further validation of the considered grey-area mitigation tools in more complex industrial flows.

\section{Acknowledgements}

The research leading to these results has received funding from the European Union Seventh Framework Programme FP7/20072013 within the project Go4Hybrid ("Grey Area Mitigation for Hybrid RANS-LES Methods") under grant agreement no. 605361. The authors from St.-Petersburg Polytechnic University thank Supercomputer Center "Polytechnichesky" for the resources of cluster Tornado, on which the NTS simulations were performed.

\section{References}

Bachalo, W.D., Johnson, D.A., 1986. Transonic, turbulent boundary-layer separation generated on an axisymmetric flow model. AIAA J. 24 (3), 437-443.

Bechara, W., Bailly, C., Lafon, P., Candel, S., 1994. Stochastic approach to noise modeling for free turbulent flows. AIAA J. 32, 455-463.

Billson, M., Eriksson, L.-E., Davidson, L., 2003. Jet noise prediction using stochastic turbulence modeling. AIAA Paper No. 2003-3282.

Greenblatt, D., Paschal, K.B., Yao, C.-S., Harris, J., Schaeffler, N.W., Washburn, A.E. 2006. Experimental investigation of separation control part 1: baseline and steady suction. AIAA J. 12, 2820-2830.

Guseva, E.K., Garbaruk, A.V., Strelets, M.Kh., 2017. Assessment of delayed DES and improved delayed DES combined with a shear-layer-adapted subgrid length-scale in separated flows. Flow Turbul. Combust. 98 (2), 481-502. 
Jarrin, N., Prosser, R., Uribe, J., Benhamadouche, S., Laurence, D., 2009. Reconstruction of turbulent fluctuations for hybrid RANS/LES simulations using a synthetic-eddy method. Int. J. Heat Fluid Flow 30, 435-442.

Kraichnan, R., 1970. Diffusion by a random velocity field. Phys. Fluids 13, 22-31.

Löwe, J., Probst, A., Knopp, T., Kessler, R., 2016. Low-dissipation low-dispersion second-order scheme for unstructured finite-volume flow solvers. AIAA J. 54 (10), 2961-2971.

Menter, F.R., 1994. Two-equation eddy-viscosity turbulence models for engineering applications. AIAA J. 32 (10), 1598-1605.

Mockett, C., Haase, W., Thiele, F., 2015a. Go4Hybrid: a European initiative for improved hybrid RANS-LES modelling. Progress in Hybrid RANS-LES Modelling. Notes Numer. Fluid Mech. Multidiscip. Des. 130, 299-303.

Mockett, C., Fuchs, M., Garbaruk, A., Shur, M., Spalart, P., Strelets, M., Thiele, F., 2015b. Two non-zonal approaches to accelerate RANS to LES transition of free shear layers in DES. Notes Numer. Fluid Mech. Multidiscip. Des. 130, 187201.

Poletto, R. Revell, A, Craft, T, Jarrin, N., 2011. Divergence free synthetic eddy method for embedded LES inflow boundary conditions. Seventh International Symposium on Turbulence and Shear Flow Phenomena (TSFP-7), Ottawa, Canada.

Probst, A., Löwe, J., Reuß, S., Knopp, T., Kessler, R., 2016a. Scale-resolving simulations with a low-dissipation low-dispersion second-order scheme for unstructured flow solvers. AIAA J. 54 (10), 2972-2987.

Probst, A., 2016b. Implementation and assessment of the synthetic-eddy method in an unstructured compressible flow solver. 6th Symposium on Hybrid RANS-LES Methods, Strasbourg, France, 26-28 September 2016.

Rogers, S.E., Kwak, D., 1988. An upwind differencing scheme for the time-accurate incompressible Navier-Stokes equations. AIAA Paper No. 88-2583.

Schwamborn, D., Gardner, A., von Geyr, H., Krumbein, A., Lüdeke, H., Stürmer, A., 2008. Development of the TAU code for aerospace applications. 50th NAL International Conference on Aerospace Science \& Technology, 26-28 June 2008, Bangalore.

Schwamborn, D., Strelets, M., 2012. ATAAC - an EU-project dedicated to hybrid RANS/LES methods. Progress in hybrid RANS-LES modelling. Notes Numer. Fluid Mech. Multidiscip. Des. 117, 59-75.
Shur, M., Strelets, M., Travin, A., 2004. High-order implicit multi-block NavierStokes code: ten-year experience of application to RANS/DES/LES /DNS of turbulent flows. 7th Symposium on Overset Composite Grids \& Solution Technology, Huntington Beach, CA http://cfd.spbstu.ru/agarbaruk/c/documentlibrary/ DLFE-42505.pdf.

Shur, M.L., Spalart, P.R., Strelets, M.Kh., Travin, A.K., 2008. A hybrid RANS-LES approach with delayed-DES and wall-modelled LES capabilities. Int. J. Heat Fluid Flow 29, 1638-1649.

Shur, M.L., Spalart, P.R., Strelets, M.Kh, Travin, A.K., 2014. Synthetic turbulence generators for RANS-LES interfaces in zonal simulations of aerodynamic and aeroacoustic problems. Flow Turbul. Combust. 93, 63-92.

Shur, M.L., Spalart, P.R., Strelets, M.Kh., Travin, A.K., 2015. An enhanced version of DES with rapid transition from RANS to LES in separated flows. Flow Turbul. Combust. 95, 709-737.

Spalart, P.R., Allmaras, S.R., 1994. A one-equation turbulence model for aerodynamic flows. La Recherche Aerospatiale 1 (1), 5-21.

Smirnov, A., Shi, S., Celik, I., 2001. Random flow generation technique for large eddy simulations and particle-dynamics modeling. J. Fluids Eng. 123 (2), 359-371.

Spalart, P.R., Deck, S., Shur, M.L., Squires, K.D., Strelets, M.Kh., Travin, A.K., 2006. New version of detached-eddy simulation, resistant to ambiguous grid densities. Theor. Comput. Fluid Dyn. 20 (3), 181-195.

Spalart, P., Belyaev, K., Garbaruk, A., Shur, M., Strelets, M., Travin, A., 2016. Large-eddy and direct numerical simulations of the Bachalo-Johnson flow with shock-induced separation. In: Proceedings of 11th International ERCOFTAC Symposium on Engineering Turbulence Modelling and Measurements, Palermo, Italy, 2016.

Travin, A., Shur, M., Spalart, P., Strelets, M., 2002. Physical and numerical upgrades in the detached-eddy simulation of complex turbulent flows. Fluid Mech. Appl. $65,239-254$.

Uzun, A., Malik, M.R., 2017. Wall-resolved large-eddy simulation of flow separation over NASA wall-mounted hump. AIAA Paper No. 2017-0538. 